\title{
O Processo de Distritalização e a Utilização de Serviços de Saúde - Avaliação do Caso de Pau da Lima, Salvador, Bahia, Brasil'1
}

\author{
District Allocation and Utilization of Health Care Services in Pau da Lima, \\ Salvador, Bahia, Brazil
}

Lígia Maria V. da Silva² ; Vera Lúcia A. Formigli2; Macius P. Cerqueira²; Leonardo Kruchevsky; Márcia Maria A. Teixeira²; Antonio Sergio M. Barbosa²; Paulo Sergio de A. Conceiçãoº; Mauro de A. Khouri \& Carlito L. Nascimento ${ }^{2}$

SILVA, LM. V.; FORMIGLI, V. LA.; CERQUEIRA, M. P.; KRUCHEVSKY, L; TEIXEIRA, M. M. A.; BARBOSA, A. S. M.; CONCEIÇÃO, P. S. A.; KHOURI, M. A. \& NASCIMENTO, C. L. District Allocation and Utilization of Health Care Services in Pau da Lima, Salvador, Bahia, Brazil. Cad. Saúde Públ., Rio de Janeiro, 11 (1): 72-84, Jan/Mar, 1995.

This cross-sectional study is aimed at the identication of patterns in the utilization of health care services in Pau da Lima, a neighborhood in the city of Salvador, Bahia. In 1992, a household survey was carried out with 384 families selected through a random cluster sampling design. In each household, the family head or a surrogate informant was asked to answer a questionnaire about their use of any type of health care in the last month. From the total of 1,887 individuals, 236 reported at least one visit to health facilities during the referent period This means an estimated prevalence of health services utilization of $12.5 \%$. With regard to the type of services, $25.9 \%$ of all visits were made to public sector facilities. Attendants were mainly women (73.7\%) from 15 to 29 years of age. The main reason reported for the choice of the health facility was availability (63.7\%), and the major reason for seeking health care was disease-related conditions (75.7\%), rather than preventive procedures. These findings may result from the still unfinished process of distritalização (district allocation of services), which is evident in the lack of health services' infrastructure or coverage, as well as in persistent organizational problems. These issues may lead the population living in the respective district to seek health care outside the assigned catchment area. The authors discuss these findings in the context of the expansion of the private sector in the health area and the financial collapse of the public budget in Brazil.

Key words: Health Services; Decentralization; Private Health Services; Public Health

\section{INTRODUÇÃO}

No Brasil, a descentralização das ações de saúde tem sido identificada como componente relevante para a gestão dos serviços desde a III Conferência Nacional de Saúde, em 1960,

\footnotetext{
'Trabalho realizado com o auxílio do Conselho Nacional de Desenvolvimento Científico e Tecnológico $(\mathrm{CNPq})$.

2 Departamento de Medicina Preventiva. Universidade Federal da Bahia. Rua Padre Feijó 29. $4^{\circ}$ andar, Anexo II, Salvador, BA, 40110-170, Brasil.
}

(Nascimento, 1991), tendo sido incorporada na Constituição de 1988 como uma das diretrizes para a organização do Sistema Único de Saúde (SUS). Posteriormente, verificou-se certo grau de institucionalização dessa diretriz a partir de iniciativas de municipalização e de distritalização, ocorridas sobretudo depois de 1985 (Silva, 1990).

Sob o rótulo de municipalização, existem concepções e práticas diferenciadas de gestão e organização de ações de saúde. Na maioria das situações, a descentralização tem ocorrido, reproduzindo o modelo assistencial dominante, de 
compra de serviços, o que corresponde a "inampização" dos sistemas municipais de saúde (Mendes, 1991). Em menor escala, têm sido desenvolvidos projetos de reorganização de práticas de saúde denominados, em algumas circunstâncias, projetos de distritalização ou de municipalização e, em outras, Sistemas Locais de Saúde (Silos) (Paim, 1993).

A avaliação desses projetos constitui-se numa necessidade relacionada com a aferição do papel dessa estratégia, seu significado e repercussões frente ao desafio de reorganização dos serviços de saúde. Nessa perspectiva, existem relatos das modificações introduzidas tanto em relação ao processo gerencial e organizacional, quanto no que concerne à atenção à saúde (Neves, 1986; Almeida et al., 1989; Formigli et al., 1992; Moraes \& Kalil, 1993). Além disso, alguns trabalhos têm comparado experiências de diversos países a partir de um conjunto de indicadores selecionados (OPS/ OMS, 1990). Outros têm recorrido à análise da cobertura assistencial (Fernandes Silva et al., 1991) ou buscado comparar a situação de saúde do período anterior à descentralização com aquela do período subseqüente à intervenção realizada, mediante análise do comportamento de atores, de problemas e de séries temporais de indicadores de serviços e de morbimortalidade (Silva, 1990). Menos freqüentes têm sido os estudos de avaliação de impacto (Unicef/Socep/SES-CE, 1990).

A compreensão do significado das experiências de descentralização requer a adoção de estratégias e técnicas de avaliação capazes de dar conta das múltiplas dimensões envolvidas no processo de reorganização dos serviços e ações de saúde de um distrito sanitário ou de um município, que, entre outros aspectos, dizem respeito aos seguintes atributos:

“a) relacionados com a disponibilidade $e$ distribuição social dos recursos (cobertura, acessibilidade e eqüidade); b) relacionados com o efeito das ações e práticas de saúde implementadas (eficácia, efetividade e impacto); c) relacionados com os custos das ações (eficiência); d) relacionados com a adequação das ações ao conhecimento técnico e científico vigente (qualidade técnico-científica); e) relacionados à percepção dos usuários sobre as práticas (satisfação dos usuários, aceitabilidade)", (Silva \& Formigli, 1994: 81).

A avaliação da disponibilidade e distribuição social dos recursos (cobertura, acessibilidade e eqüidade) é um pré-requisito para a avaliação dos demais atributos, tendo em vista que, para se discutir qualidade, impacto e satisfação, é preciso que as ações de saúde sejam preliminarmente oferecidas à população usuária. Nesse caso, dados obtidos a partir do registro rotineiro das unidades têm sido utilizados mais freqüentemente para o cálculo de indicadores de concentração de consultas ou de cobertura assistencial. O recurso a estudos de utilização de serviços de base populacional apresenta vantagens, tendo em vista não só a possibilidade de se aferir a demanda espontânea real e a utilização dos serviços programados de forma organizada, como obter informações acerca daquele segmento que não é usuário dos serviços de saúde estudados (Carvalho et al., 1988). As dificuldades residem na incorporação de inquéritos populacionais na rotina da avaliação de distritos sanitários, devido aos cultos e complexidade desse tipo de estudo.

O Distrito Sanitário de Pau da Lima, organizado em 1988, vem buscando transformar o modelo assistencial a partir da introdução de planejamento situacional, de democratizado da gestão e de modificações na organização dos serviços (Formigli et al., 1992; Kadt \& Tasca, 1993).

Este estudo tem por objetivo avaliar o padrão de utilização dos serviços de saúde, bem como obter informações acerca da satisfação dos usuários a partir de inquérito populacional nesse distrito sanitário. Faz parte de um projeto mais amplo que busca desenvolver estratégia metodológica para avaliação de projetos de reorganização de práticas de saúde em nível local (Silva \& Formigli, 1993), ou seja, a avaliação de um distrito não se reduz ao estudo da disponibilidade e adequação dos recursos, que é um dos seus componentes. Por sua vez, o estudo da utilização dos serviços de saúde é apenas uma das técnicas possíveis de serem acionadas com essa finalidade. 


\section{METODOLOGIA}

Foi realizado estudo transversal conduzido com base em inquérito domiciliar no Distrito Sanitário de Pau da Lima, através do recurso a técnica de amostragem por conglomerados. Essa técnica vem sendo recomendado pela Organização Mundial de Saúde, por meio do seu Programa Ampliado de Imunizações (PAI) para a aferição da cobertura vacinal (Lemeshow \& Robinson, 1983). Dessa forma, tem sido utilizado com razoável sucesso não só com essa finalidade como também para avaliar necessidades de serviços de saúde em diversos países. Além disso, validação feita por meio de simulações em computador (Henderson \& Sundaresan, 1982) apontam vantagens e a viabilidade de sua incorporação na rotina dos serviços pela simplicidade de sua execução.

Optou-se pela definição de um erro de 0,05, menor do que aquele proposto pelo Programa Ampliado de Imunizações, de 0,10. O tamanho da amostra, de 384 domicílios, foi obtido na, tabela de manual da Organização Mundial da Saúde (OMS) referente aos estudos em que se busca a estimativa de proporção populacional, com precisão absoluta especificada (Lwanga \& Lemeshow, 1991).

Foram sorteadas 30 microáreas da lista das 50 identificadas pela gerência do Distrito (Kadt \& Taska, 1993). Em cada microárea foi sorteada uma rua, da qual foram visitados 13 domicílios em seqüência até que o tamanho da amostra fosse atingido. Nas microáreas referentes a favelas ou invasões, onde não havia ruas, iniciou-se pelo primeiro domicílio encontrado, continuando as entrevistas naqueles subseqüentes até completar 13. Em cada domicílio, considerado como unidade amostral, foram entrevistados todos os usuários que utilizaram serviços de saúde no mês anterior à data da entrevista. $\mathrm{Na}$ ausência de usuário, as informações foram obtidas junto ao membro da família presente no domicílio no momento da entrevista. Nas entrevistas buscou-se a investigação de todas as utilizações de serviços ocorridas no mês anterior à coleta dos dados. Considerou-se como utilização de serviço de saúde a procura de centro de saúde, hospital ou unidade de emergência com a finalidade de submeter-se tanto a ações curativas (consulta médica ou odontológica) como a ações preventivas (imunização, pré-natal, puericultura e outros). Foram ainda investigadas as seguintes variáveis: a) tipo de serviço utilizado (público do distrito; privado do distrito; público fora do distrito e privado fora do distrito); b) motivo para a procura (curativo, preventivo, obstétrico-ginecológico); c) razões para a não-utilização dos serviços públicos do Distrito; d) razões para a escolha do serviço utilizado. O motivo de o atendimento obstétrico-ginecológico ter sido considerado em separado relaciona-se com o fato de que essa especialidade envolve ações preventivas e curativas. Foram acrescentadas, ainda, três questões sobre a satisfação dos usuários, tendo em vista a importância desse atributo para a avaliação global do sistema.

Foi realizado pré-teste em 27/10/92, que resultou em modificação do questionário. A coleta de dados ocorreu nos meses de novembro e dezembro de 1992. Os dados foram digitados e consolidados no programa EPI-INFO. Foi calculada a taxa global de utilização - frequiência de utilização - por tipo de serviço de saúde, por faixa etária e por sexo. Os motivos da escolha do tipo de serviço e as razões para a não-utilização dos serviços do Distrito Sanitário de Pau da Lima foram reagrupados, e as frequiências, calculadas. Para análise das perguntas relacionadas com a satisfação dos usuários, foram calculadas razões entre as respostas favoráveis (totalmente resolvido, parcialmente resolvido, ficou satisfeito, atendimento excelente e bom) e aquelas desfavoráveis (não resolveu, piorou, não ficou satisfeito, atendimento ruim e péssimo).

\section{DISCUSSÃO DOS RESULTADOS}

\section{Utilização e Cobertura Assistencial}

Entre as 1887 pessoas moradoras dos domicílios visitados, 251 haviam procurado serviços de saúde nos últimos 30 dias. Destes, 236 utilizaram de fato os serviços, o que corresponde à taxa de utilização de $12,5 \%$ e à demanda de $13,3 \%$. Estudos realizados no Estado da Bahia entre 1982 e 1986 revelaram variação entre 4,9\% (Subaúma) e 15,2\% (Camaçari) da taxa de utilização de consultas médicas nos 15 dias anteriores (Carvalho et al., 1988). Na Pesquisa Nacional de Amostra 
Domiciliar (PNAD) da Fundação Instituto Brasileiro de Geografia e Estatística (IBGE), de 1981, a proporção de pessoas que haviam utilizado serviços de saúde nos 30 dias anteriores foi de 7,7\%, para o Brasil, e de 4,8\%, para a região Nordeste (Simões, 1981). Já na PNAD de 1986, a taxa encontrada para os 15 dias anteriores, para a Bahia, foi de 9,2\%. A taxa encontrada no Distrito Sanitário de Pau da Lima situa-se na faixa de variação registrada nos diversos estados; a diversidade das metodologias empregadas, entretanto, dificulta a comparação.

Supondo que a taxa encontrada corresponda a um valor médio, pode-se estimar a demanda potencial para utilização dos serviços de saúde, nessa área, em 237.348 atendimentos/ ano ou 1,6 atendimentos por habitante/ano, tomando por base a estimativa populacional do IBGE, de 148.715 habitantes para 1992. Do total de episódios de utilização registrados, $67,4 \%$ podem ser considerados geradores de consultas médicas (utilização por doença, acidente e atendimento ginecológico), o que corresponde à demanda potencial de 159.973 consultas médicas/ano ou 1,1 consultas médicas/ habitante/ano.

O Distrito Sanitário de Pau da Lima produziu em 1991 o total de 192.872 consultas médicas, sendo 53.700 em unidades públicas e $139.172 \mathrm{em}$ privadas, o que corresponde à concentração de consultas médicas de 1,3/hab/ano e à cobertura de $120 \%$. A capacidade instalada no Distrito parece, portanto, ser suficiente para atender à demanda espontânea da população.
A análise da adequação quantitativa dos serviços produzidos pelo distrito em relação à demanda espontânea da população pode ser feita a partir da comparação entre a concentração de consultas médicas e três padrões distintos: a demanda espontânea calculada a partir do estudo de utilização de base populacional; o padrão do Conasp (Conselho Consultivo de Administração de Saúde Previdenciária), de duas cons/hab/ano (MPAS/Conasp, 1982) e o padrão da OMS, que prevê entre três e quatro cons/hab/ano (OMS, 1981). A proporção de adequação ou cobertura dos serviços públicos do distrito assim calculada seria de $33,6 \%, 18 \%$ e $12 \%$, respectivamente. Já os serviços privados do distrito apresentam cobertura potencial de $86,9 \%, 46,8 \%$ e $31,2 \%$, ou seja, a despeito da ampliado na produção de serviços verificada com a organização do Distrito Sanitário em Pau da Lima (SUS/DSPL, 1992), verificou-se ainda insuficiência quantitativa desses recursos, mesmo em relação ao atendimento da demanda espontânea, na hipótese de os serviços públicos do distrito passarem a ser isoladamente responsáveis pela atenção à saúde naquela área. Chama atenção a diferença obtida a partir da utilização dos diferentes padrões, o que evidencia a fragilidade desse indicador como estimador da cobertura assistencial.

A análise da Tabela 1 revela que os serviços públicos do Distrito Sanitário foram responsáveis por apenas $25,9 \%$ do total dos atendimentos, enquanto as demais unidades atenderam a 74,1\%

TABELA 1. Utilização por Tipo de Serviço, Distrito Sanitário de Pau da Lima, Salvador, Bahia, 1992

\begin{tabular}{lcc}
\hline \hline & \multicolumn{2}{c}{ Utilização } \\
\cline { 2 - 3 } Tipo de Serviço & № & $\%$ \\
\hline Público do Distrito & 65 & 25,9 \\
Público Fora do Distrito & 74 & 29,5 \\
Particular do Distrito & 49 & 19,5 \\
Particular Fora do Distrito & 39 & 15,5 \\
Outros & 20 & 8,0 \\
Sem Informação & 4 & 1,6 \\
\hline Total & 251 & 100,0 \\
\hline \hline
\end{tabular}


da demanda espontânea da população. Essa baixa utilização não se relaciona nem com a capacidade instalada, nem com deficiências de recursos humanos, pois existe relativa ociosidade nas unidades públicas do distrito, principalmente no período vespertino, decorrente da baixa produtividade, que varia entre $35,0 \%$ e 63,4\% (SUS/DSPL, 1993). A despeito de o distrito ter elevação tanto a produção global de serviços quanto a produtividade entre 1988 e 1992 (SUS/DSPL, 1993), a cobertura assistencial é ainda insuficiente para atender à demanda espontânea da população.

O simples cálculo da concentração de consultas, embora possa permitir aferição aproximada da adequação quantitativa de recursos, deixa de revelar a forma concreta pela qual esses serviços estão sendo de fato utilizados. Por exemplo, este estudo revela que, a população utilizava quase igualmente os serviços situados dentro do território do distrito $(45,4 \%)$ em relação aqueles situados fora $(48,6 \%)$ (se incluirmos os serviços classificados como "outros", situados fora do distrito). Por outro lado, a utilização dos serviços públicos $(55,4 \%)$ foi superior aquela dos privados (35\%), embora entre os públicos a preferência tivesse sido para os serviços situados fora do distrito (Tabela 1). Esse achado revela um estágio incipiente no alcance de um dos objetivos da proposta de distritalização, que é o fato de a população reconhecer os serviços como "seus" (MPAS/ Inamps, 1987) e, conseqüentemente, priorizá-los na procura.
Por último, a distribuição da utilização de serviços segundo a faixa etária revelou percentual maior entre 15 e 29 anos (29,8\%), semelhante aquele encontrado na PNAD de 1986, em que $22,8 \%$ das utilizações de serviços se encontrava nesse grupo etário (IBGE, 1986). Em ambos os estudos, a menor utilização foi no grupo de menores de um ano.

Em relação à distribuição por sexo, verificou-se predominância da utilização por parte do sexo feminino $(73,7 \%)$, resultado que coincide com os achados de outros estudos (IBGE, 1986; Carvalho et al., 1988).

\section{Tipo de Serviço Utilizado: As Razões da Escolha}

Os principais motivos para a escolha do serviços de saúde relacionaram-se com a acessibilidade (Tabela 2). A proximidade da residência ou local de trabalho (acesso geográfico) foi o motivo que, isoladamente, se associou à maior proporção de escolhas por parte dos usuários $(35,4 \%)$. Quando se somam outros motivos correlatos, como "direito ao serviço ou convênio" (7,2\%), "referência/indicação" (5,6\%) e "facilidades/relações pessoais" (2,8\%), que podem ser considerados expressões da acessibilidade econômica e cultural, esse conjunto de razões responde por $51 \%$ do total. Em seguida, situam-se motivos relacionados com características do

TABELA 2. Razões para a Escolha do Serviço de Saúde, Distrito Sanitário de Pau da Lima, Salvador, Bahia, 1992

\begin{tabular}{lcc}
\hline \hline Razão da Escolha do Serviços de Saúde & $\mathrm{N}^{\mathbf{0}}$ & $\%$ \\
\hline Proximidade da residência ou trabalho/Facilidade de transporte & 89 & 35,4 \\
Rapidez no atendimento & 32 & 12,7 \\
Bons profissionais & 25 & 9,9 \\
Capacidade de resolver o problema & 20 & 8,0 \\
Direito ao serviço/Convênios & 18 & 7,2 \\
Referência/Indicação & 14 & 5,6 \\
Oferta de serviços demandados & 12 & 4,8 \\
Facilidades/Relações pessoais & 07 & 2,8 \\
Qualificação positiva do serviço & 05 & 2,0 \\
Outros & 12 & 4,8 \\
Não-obtido & 17 & 6,8 \\
\hline Total & 251 & 100,0 \\
\hline \hline
\end{tabular}


serviço e da atenção à saúde propriamente dita, como "rapidez no atendimento" (12,7\%), "presença de bons profissionais" $(9,9 \%)$, "capacidade de resolver o problema" (8\%), "qualificação positiva do serviço" (2\%), que perfazem o total de $32,6 \%$. A “oferta de serviços demandados" (4,8\%), pode ser considerada característica do serviço, relacionada tanto com disponibilidade e adequação da atenção quanto com acessibilidade. Esse peso maior dos fatores relativos ao acesso, em detrimento daqueles decorrentes das características do cuidado, ainda que a partir do ponto de vista subjetivo do usuário, revela a persistência de problemas relacionados com a organização dos serviços e sua adequação quantitativa.

Também, quando é investigado o motivo da não-utilização dos serviços do distrito sanitário, observa-se que ganham relevo aqueles relacionados com a oferta e a possibilidade de utilização dos serviços por parte da população, que, segundo Frenk (1985), se constituem em componentes da acessibilidade. Assim, além da "dificuldade de acesso" claramente explicitada $(9,7 \%)$, a "falta de profissionais" $(18,3 \%)$, a "inexistência do serviços demandado" $(7 \%)$ e a "demora no atendimento" $(24,7 \%)$ podem constituir-se em indicadores das dificuldades encontradas pelos usuários para a utilização dos serviços.
Outras características do serviço como aspectos referentes à relação interpessoal de profissionais e população, como "falta de atenção aos doentes" $(2,7 \%)$ e "ausência de bons profissionais" $(2,1 \%)$, passam para um segundo plano das alegações dos usuários para a não-opção pelos serviços do distrito (Tabela 3).

O grupo dos "outros motivos" (16,7\%) engloba, entre outras justificativas, algumas alegações genéricas e subjetivas, negativas em relação aos serviços do distrito tais, como "- Não levo fé no Posto..." ou ainda “- O serviço não é confiável" (sic). Chama atenção o percentual de $13,4 \%$ entre os entrevistados que referiram desconhecer os referidos serviços.

Tais achados indicam que as insuficiências e deficiências dos serviços públicos do distrito limitam a sua escolha por parte da população.

\section{O Motivo da Procura e a Oferta Organizada de Ações}

A demanda aos serviços de saúde públicos do distrito decorreu fundamentalmente de condições relacionadas com a ocorrência de doença e a busca de serviços curativos $(75,7 \%)$, em contraposição com a utilização de serviços preventivos, como imunização, puericultura e pré-natal $(16,7 \%)$. Da mesma forma, os serviços públicos

TABELA 3. Razões para a Não-Utilização dos Serviços Públicos do Distrito, Distrito Sanitário de Pau da Lima, Salvador, Bahia, 1992

\begin{tabular}{lrr}
\hline \hline Razão para a Não-Utilização & & \\
dos Serviços Públicos & $\mathrm{N}^{\mathrm{o}}$ & $\%$ \\
do Distrito Sanitário da Pau de Lima & 46 & 24,7 \\
\hline Demora no atendimento & 34 & 18,3 \\
Falta de profissionais & 25 & 13,4 \\
Desconhecimento sobre os serviço & 18 & 9,7 \\
Acesso Difícil & 13 & 7,0 \\
Inexistência de oferta do serviços demandado & 05 & 2,7 \\
Falta de atenção aos doentes & 04 & 2,1 \\
Ausência de bons profissionais & 31 & 16,7 \\
Outros & 10 & 5,4 \\
Não obtidos & 186 & 100,0 \\
\hline Total
\end{tabular}


externos ao distrito foram procurados pelos mesmos motivos: $66,2 \%$ e $21,6 \%$, respectivamente (Tabela 4). Esses achados são compatíveis com aqueles da PNAD/1986 do IBGE, em que as ações curativas responderam por $82,8 \%$ das utilizações, e as ações preventivas, por 13,7\%. São resultados que revelam a persistência do modelo assistencial dominante, centrado no atendimento médico-hospitalar, curativo, e na demanda espontânea dos serviços. O Distrito Sanitário de Pau da Lima tem desenvolvido iniciativas voltadas para a organizado da oferta dos serviços, principalmente no que diz respeito à reorientação da própria demanda espontânea em algumas unidades de saúde para as ações do Programa de Atenção à Saúde da Criança (Paisc) e do Programa de Atenção à saúde da Mulher (Paism). Essas ações, contudo, ainda não têm expressão quantitativa possível de ser identificada por meio de estudo desse tipo. Outras medidas introduzidas, como a busca de sintomáticos respiratórios e a articulação entre ações de saneamento e saúde, são mais recentes e incipientes ainda, algumas delas introduzidas em período posterior ao da realização da coleta de dados do presente estudo.

Também o fato de a utilização ser pequena entre menores de um ano $(4,4 \%)$ revela que a prioridade para o controle dos problemas da infância ainda não foi operacionalizada. $\mathrm{O}$ fato de a maior parte dos usuários ser constituída por mulheres $(73,7 \%)$ na faixa etária entre $15 \mathrm{e}$ 29 anos $(29,8 \%)$ indica que problemas de saúde de magnitude, como a hipertensão arterial e a tuberculose, também prevalentes em homens, não estão demandando atendimento nas unidades nem sendo identificados a partir de ações do distrito.

TABELA 4. Motivos para a Procura por Tipo de Serviço Público, Distrito Sanitário de Pau da Lima, Salvador, Bahia, 1992

\begin{tabular}{|c|c|c|c|c|c|}
\hline \multirow{3}{*}{$\begin{array}{l}\text { Motivo } \\
\text { da } \\
\text { Procura }\end{array}$} & \multicolumn{4}{|c|}{ Tipo de Serviço } & \multirow{3}{*}{ Total } \\
\hline & \multicolumn{2}{|c|}{$\begin{array}{l}\text { Serviço Público do } \\
\text { Distrito Sanitário de } \\
\text { Pau de Lima }\end{array}$} & \multicolumn{2}{|c|}{$\begin{array}{c}\text { Serviço Público de Fora do } \\
\text { Distrito Sanitário de Pau } \\
\text { da Lima }\end{array}$} & \\
\hline & $\mathrm{N}^{\mathrm{o}}$ & $\%$ & $\mathrm{~N}^{\mathrm{o}}$ & $\%$ & \\
\hline Curativo & 50 & 75,7 & 49 & 66,2 & 99 \\
\hline Preventivo & 11 & 16,7 & 16 & 21,6 & 27 \\
\hline Obstétrico/Ginecológico & 05 & 7,6 & 09 & 12,2 & 14 \\
\hline Total & 66 & 100 & 74 & 100 & 140 \\
\hline
\end{tabular}

\section{A Satisfação e a Resolução do Problema na Perspectiva do Usuário}

Verificou-se elevada proporção de entrevistados $(82,2 \%)$ que informaram estar satisfeitos com o atendimento recebido (Tabela 5). Também a avaliação positiva (excelente e bom) do serviço correspondeu a 64,0\% dos usuários (Tabela 6). Houve ainda concordância entre essas respostas e a percepção por parte de $78,8 \%$ dos mesmos de que seu problema havia sido total ou parcialmente resolvido (Tabela 7).

Quando a resposta a essas três questões é analisada por tipo de serviços, observa-se que, entre os cinco grupos de serviços utilizados, os públicos do distrito apresentam as menores razões entre o total de respostas classificadas como satisfatórias e insatisfatórias (Tabela 5; Tabela 6 e Tabela 7). Chama atenção o fato de que apenas em relação ao grupo de serviços do distrito tenha havido três referencias à piora do problema.

Assim, a análise da satisfação por tipo de serviços foi desfavorável aos serviços públicos do distrito sanitário. A razão respostas satisfatórias/respostas não-satisfatórias foi 21,5 para os serviços privados localizados no território do distrito; 10,3 para o serviços privados externos ao distrito; 6,4 para os serviços públicos exter- 
TABELA 5. Satisfação do Usuário por Tipo de Serviço, Distrito de Pau da Lima, Salvador, Bahia

\begin{tabular}{|c|c|c|c|c|c|c|c|c|c|c|c|c|c|c|}
\hline \multirow{4}{*}{$\begin{array}{l}\text { Ficou } \\
\text { Satisfeito }\end{array}$} & \multicolumn{14}{|c|}{ Tipo de Serviço } \\
\hline & \multirow{2}{*}{\multicolumn{2}{|c|}{$\begin{array}{l}\text { Público } \\
\text { Distrito }\end{array}$}} & \multirow{2}{*}{\multicolumn{2}{|c|}{$\begin{array}{l}\text { Privado } \\
\text { Distrito }\end{array}$}} & \multirow{2}{*}{\multicolumn{2}{|c|}{$\begin{array}{c}\text { Público } \\
\text { Fora }\end{array}$}} & \multirow{2}{*}{\multicolumn{2}{|c|}{$\begin{array}{c}\text { Privado } \\
\text { Fora }\end{array}$}} & \multicolumn{6}{|c|}{ Sem } \\
\hline & & & & & & & & & \multicolumn{2}{|c|}{ Outros } & \multicolumn{2}{|c|}{ Informação } & \multicolumn{2}{|c|}{ Total } \\
\hline & № & $\%$ & № & $\%$ & № & $\%$ & № & $\%$ & № & $\%$ & № & $\%$ & № & $\%$ \\
\hline $\operatorname{Sim}(A)$ & 41 & 74,5 & 43 & 89,6 & 58 & 80,6 & 31 & 83,8 & 20 & 100 & 01 & 25 & 194 & 82,2 \\
\hline Não (B) & 09 & 16,4 & 02 & 4,2 & 09 & 12,5 & 03 & 8,1 & 00 & - & 00 & - & 23 & 9,7 \\
\hline Em Termos & 03 & 5,5 & 00 & - & 05 & 6,9 & 00 & - & 00 & - & 00 & - & 08 & 3,4 \\
\hline Sem Informação & 02 & 3,6 & 03 & 6,2 & 00 & - & 03 & 8,1 & 00 & - & 03 & - & 11 & 4,7 \\
\hline Total & 55 & 100,0 & 48 & 100,0 & 72 & 100,0 & 37 & 100,0 & 20 & 100,0 & 04 & 100,0 & 236 & 100,0 \\
\hline Razão A/B & \multicolumn{2}{|c|}{4,5} & \multicolumn{2}{|c|}{21,5} & \multicolumn{2}{|c|}{6,4} & \multicolumn{2}{|c|}{10,3} & \multicolumn{2}{|c|}{ - } & \multicolumn{2}{|c|}{-} & \multicolumn{2}{|c|}{-} \\
\hline
\end{tabular}

TABELA 6. Avaliação do Atendimento pelos Usuários por Tipo de Serviço, Distrito de Pau da Lima, Salvador, Bahia, 1992

\begin{tabular}{|c|c|c|c|c|c|c|c|c|c|c|c|c|c|c|}
\hline \multirow{4}{*}{$\begin{array}{l}\text { Avaliação do } \\
\text { atendimento }\end{array}$} & \multicolumn{14}{|c|}{ Tipo de Serviço } \\
\hline & \multirow{2}{*}{\multicolumn{2}{|c|}{$\begin{array}{l}\text { Público } \\
\text { Distrito }\end{array}$}} & \multirow{2}{*}{\multicolumn{2}{|c|}{$\begin{array}{l}\text { Privado } \\
\text { Distrito }\end{array}$}} & \multirow{2}{*}{\multicolumn{2}{|c|}{$\begin{array}{c}\text { Público } \\
\text { Fora }\end{array}$}} & \multirow{2}{*}{\multicolumn{2}{|c|}{$\begin{array}{c}\text { Privado } \\
\text { Fora }\end{array}$}} & \multicolumn{6}{|c|}{ Sem } \\
\hline & & & & & & & & & \multicolumn{2}{|c|}{ Outros } & \multicolumn{2}{|c|}{ Informação } & \multicolumn{2}{|c|}{ Total } \\
\hline & $\mathrm{N}^{\mathrm{o}}$ & $\%$ & № & $\%$ & № & $\%$ & № & $\%$ & № & $\%$ & № & $\%$ & № & $\%$ \\
\hline Péssimo (A) & 04 & 7,3 & 01 & 2,1 & 05 & 6,9 & 00 & - & 00 & - & 00 & - & 10 & 4,2 \\
\hline Ruim (B) & 04 & 7,3 & 02 & 4,2 & 04 & 5,6 & 02 & 5,4 & 00 & - & 00 & - & 12 & 5,1 \\
\hline Regular & 11 & 20 & 08 & 16,7 & 19 & 26,4 & 06 & 16,2 & 01 & 5 & 01 & 25 & 46 & 19,5 \\
\hline Bom (C) & 23 & 41,8 & 21 & 43,7 & 26 & 36,1 & 13 & 35,2 & 11 & 55 & 00 & - & 94 & 39,8 \\
\hline Excelente (D) & 10 & 18,2 & 13 & 27,1 & 15 & 20,8 & 12 & 32,4 & 07 & 35 & 00 & - & 57 & 24,2 \\
\hline Sem Informação & 03 & 5,4 & 03 & 6,2 & 04 & 4,2 & 03 & 10,8 & 01 & 5 & 03 & 75 & 17 & 7,2 \\
\hline Total & 55 & 100,0 & 48 & 100,0 & 72 & 100,0 & 37 & 100,0 & 20 & 100,0 & 04 & 100,0 & 236 & 100,0 \\
\hline Razão $C+D / A+B$ & \multicolumn{2}{|c|}{4,1} & \multicolumn{2}{|c|}{11,3} & \multicolumn{2}{|c|}{4,5} & \multicolumn{2}{|c|}{12,5} & \multicolumn{2}{|c|}{-} & \multicolumn{2}{|c|}{-} & \multicolumn{2}{|c|}{-} \\
\hline
\end{tabular}


TABELA 7. Resolução do Problema segundo a Percepção do Usuário por Tipo de Serviço, Distrito Sanitário de Pau de Lima, Salvador, Bahia

\begin{tabular}{|c|c|c|c|c|c|c|c|c|c|c|c|c|c|c|}
\hline \multirow[b]{4}{*}{ OQue Aconteceu } & \multicolumn{14}{|c|}{ Tipo de Serviço } \\
\hline & \multirow{2}{*}{\multicolumn{2}{|c|}{$\begin{array}{l}\text { Público } \\
\text { Distrito }\end{array}$}} & \multirow{2}{*}{\multicolumn{2}{|c|}{$\begin{array}{l}\text { Privado } \\
\text { Distrito }\end{array}$}} & \multirow{2}{*}{\multicolumn{2}{|c|}{$\begin{array}{c}\text { Público } \\
\text { Fora }\end{array}$}} & \multirow{2}{*}{\multicolumn{2}{|c|}{$\begin{array}{c}\text { Privado } \\
\text { Fora }\end{array}$}} & \multicolumn{6}{|c|}{ Sem } \\
\hline & & & & & & & & & \multicolumn{2}{|c|}{ Outros } & \multicolumn{2}{|c|}{ Infonnação } & \multicolumn{2}{|c|}{ Total } \\
\hline & № & $\%$ & № & $\%$ & № & $\%$ & № & $\%$ & № & $\%$ & № & $\%$ & № & $\%$ \\
\hline Totalmente resolvido (A) & 23 & 41,8 & 19 & 39,6 & 37 & 51,4 & 16 & 43,3 & 14 & 70 & 01 & 25 & 110 & 46,6 \\
\hline Parcialmente resolvido (B) & 16 & 29,1 & 19 & 39,6 & 25 & 34,7 & 12 & 32,4 & 04 & 20 & 00 & - & 76 & 32,2 \\
\hline Piorou (C) & 03 & 5,5 & 00 & - & 00 & - & 00 & - & 00 & - & 00 & - & 03 & 1,3 \\
\hline Não foi resolvido (D) & 07 & 12,7 & 05 & 10,4 & 04 & 5,6 & 02 & 5,4 & 01 & 5 & 00 & - & 19 & 8,1 \\
\hline Outra alternativa & 05 & 9,1 & 03 & 6,2 & 04 & 5,6 & 05 & 13,5 & 00 & - & 00 & - & 17 & 7,2 \\
\hline Sem Informação & 01 & 1,8 & 02 & 4,2 & 02 & 2,7 & 02 & 5,4 & 01 & 5 & 03 & 75 & 11 & 4,7 \\
\hline Total & 55 & 100,0 & 48 & 100,0 & 72 & 100,0 & 37 & 100,0 & 20 & 100 & 04 & 100,0 & 236 & 100,0 \\
\hline Razão A+B/C+D & & & & & & & & & & & & - & & - \\
\hline
\end{tabular}


nos ao distrito; e apenas 4,5 para aqueles públicos do distrito (Tabela 5).

Da mesma forma, a análise da relação entre as respostas favoráveis (bom e excelente) e desfavoráveis (ruim e péssimo) relativas à avaliação feita pelos usuários por tipo de serviço revelou valores menores para os serviços públicos do distrito quando comparados com os demais tipos de serviços. Nesse caso, a ordem ascendente foi: públicos do distrito $(4,1)$; públicos externos ao distrito $(9,5)$; e privados do distrito $(11,3)$ (Tabela 6).

Quando a "resolução" do problema, segundo a percepção do usuário, foi investigada, verificou-se também maior razão entre respostas favoráveis (totalmente resolvido, parcialmente resolvido) e desfavoráveis (não foi resolvido e piorou) nos serviços públicos situados fora do distrito $(15,5)$ e naqueles privados também externos ao distrito (14) do que nos serviços privados $(7,6)$ e públicos $(3,9)$ do distrito (Tabela 7).

Os elevados percentuais de satisfação do usuário encontrados para o conjunto de serviços públicos e privados investigado merece algumas considerações diante da reconhecida precariedade desses serviços na atual conjuntura, bem como das diversas manifestações de insatisfação encontradas quase cotidianamente nos meios de comunicação de massa. A obtenção de percentuais elevados de satisfação tem sido constante em diversos serviços do país, a despeito do quadro sanitário caótico. A esse respeito, é ilustrativo o escudo realizado num hospital universitário do Rio de Janeiro, onde $90 \%$ dos atendimento médicos e $82,5 \%$ dos de enfermagem foram considerados satisfatórios pelos usuários e bastante criticados por estudantes de medicina, que identificavam falhas em diversos aspectos da organização do cuidado (Lemme et al., 1991). É possível que esses resultados favoráveis se relacionem com outros fatores decorrentes do receio por parte do usuário de perder o direito ao serviço, mesmo ele sendo precário, ou ainda com o modesto nível de exigência da população de baixa renda em relação à qualidade do serviços.

Esse tipo de resultado pode também estar relacionado com a metodologia empregada para a obtenção da informação, com o tipo de entre- vistador e com a maneira pela qual as perguntas são formuladas. Algumas estratégias metodológicas relacionadas com a investigação qualitativa (Minayo, 1992) podem ser acionadas para superar esses obstáculos. Campos (1988) sugere que os resultados obtidos nos inquéritos populacionais sejam validados por indicadores obtidos nos serviços de saúde.

Diante dessa problemática, ganham relevância os resultados desfavoráveis encontrados no distrito sanitário, ou seja, quando a tendência geral é registrar a satisfação, as indicações em contrário devem ser sobremodo valorizadas e investigadas.

\section{COMENTÁRIOS FINAIS}

A concepção que norteia os projetos de reorganização de práticas de saúde conhecidos como distritalização (Mendes, 1991; Paim, 1993) é avançada no que diz respeito às estratégias tanto de identificação de problemas de saúde quanto no que concerne às iniciativas relacionadas com sua resolução.

A avaliação desse tipo de experiência requer abordagem global que contenha informações relacionadas a modelo assistencial, cobertura, acesso, eqüidade, qualidade e impacto, devendo ser contextualizada, pois o desenvolvimento concreto das ações e práticas de saúde não depende exclusivamente das características da gestão.

Após a aferição da existência de processo de planejamento voltado para a identificação de problemas para as quais tenham sido implementadas ações pertinentes de controle, é necessário avaliar a adequação quantitativa dessas ações, a saber, a sua cobertura. O escudo da utilização dos serviços constitui-se numa das técnicas a serem acionadas, de forma complementar, em proposta global para avaliação de projetos de reorganização de práticas, que pode revelar aspectos que não emergem das informações obtidas a partir dos registros rotineiros das unidades.

Neste estudo, o fato de as variáveis relacionadas com a acessibilidade terem sido as principais responsáveis pela escolha do serviço de saúde relaciona-se com o fato de que, no nosso meio, a adequação quantitativa dos 
recursos e o acesso aos mesmos se constituem, ainda, em problema relevante em detrimento mesmo da qualidade e efetividade. No caso do Distrito Sanitário de Pau da Lima, a despeito das diversas iniciativas inovadoras implementadas (Formigli et al., 1992; SUS/DSPL, 1993), os resultados do estado revelam que o atual estágio de organização dos serviços ainda não é suficiente para que a população local os utilize de forma preferencial, como porta de entrada. Por outro lado, a predominância do atendimento curativo em detrimento do preventivo, mesmo nos serviços públicos do distrito, indica que as ações relacionadas com a oferta organizada de serviços ainda não possuem magnitude para se expressar ou que seria necessário outro tipo de técnica para avaliar a sua cobertura.

Também o fato de a população local ser predominantemente usuária de serviços públicos externos ao distrito indica que existem iniciativas a serem tomadas no âmbito do distrito capazes de atenuar parte do quadro. Nesse sentido, modificações relacionadas à divulgação dos serviços locais, ações ofertadas e horários de funcionamento, bem como a expansão das ações programáticas e atividades extramuros são requeridas. Também melhor dimensionamento dos fatores relacionados com a baixa produtividade das unidades, mencionados nos relatórios de avaliação (SUS/DSPL, 1991), pode contribuir para que o espaço de intervenção do distrito fique mais bem delimitado.

Não se pode obscurecer, contudo, a participação relevante dos serviços privados no atendimento à demanda espontânea na área, com oferta de serviços de emergência e pronto atendimento, praticamente inexistentes nas unidades públicas do distrito, bem como a significativa proporção dos entrevistados que haviam utilizado serviços de saúde e têm direito a algum tipo de assistência médica supletiva (23\%). Essa situação reflete o deslocamento, verificado na década de 80 , de parte da clientela do setor público para a assistência médica supletiva, que se expande à costa da falência do público.

Além disso, a atual conjuntura de colapso do financiamento setorial e de retrocesso em relação ao processo de reforma sanitária tem-se constituído em cenário extremamente desfavo- rável ao desenvolvimento de iniciativas concretas relacionadas com a operacionalização de propostas de distritalização.

O acompanhamento e a avaliação dessas experiências nos seus diversos aspectos é requisito para a identificação dos seus limites e possibilidades, bem como para que os obstáculos estruturais e conjunturais não comprometam a própria proposta transformadora.

\section{RESUMO}

SILVA, L. M. V.; FORMIGLI, V. L. A.; CERQUEIRA, M. P.; KRUCHEVSKY, L.; TEIXERA, M. M. A.; BARBOSA, A. S. M.; CONCEIÇÃO, P. S. A.; KHOURI, M. A. \& NASCIMENTO, C. L. O Processo de Distritalização e a Utilização de Serviços de Saúde - Avaliação do Caso de Pau da Lima, Salvador, Bahia, Brasil. Cad. Saúde Públ., Rio de Janeiro, 11 (1): 72-84, jan/mar, 1995.

Foi realizado estudo transversal de utilização de serviços de saúde no Distrito Sanitário de Pau da Lima, Salvador, Bahia, Brasil, em 1992, a partir de inquérito domiciliar com recurso à técnica de amostragem por conglomerados. Entre as 1887 pessoas moradoras dos 384 domicílios visitados, 236 haviam utilizado serviços de saúde nos últimos 30 dias, correspondendo à taxa global de utilização de $12,5 \%$. Os serviços públicos do distrito foram responsáveis por $25,9 \%$ do total dos atendimentos, tendo ocorrido maior utilização na faixa etária entre 15 e 29 anos $(29,8 \%)$ e no sexo feminino $(73,7 \%)$. A escolha do tipo de serviços foi influenciada principalmente por razões relacionadas com a acessibilidade $(63,7 \%)$. A procura dos serviços decorreu sobretudo de motivos relacionados com a ocorrência de doença e com a busca de serviços curativos $(75,7 \%)$. Esses achados revelam incipiência no processo de distritalização, expresso tanto em insuficiências quantitativas relacionadas com a cobertura assistencial e a oferta de serviços, quanto em problemas organizacionais que fazem com que a produção ainda não utilize preferencialmente os serviços do Distrito. Os autores discutem 
esses resultados no atual contexto de expansão do setor privado e de colapso de financiamento setorial.

Palavras-Chave: Serviços de Saúde;

Descentralizado; Serviços Privados de Saúde;

Saúde Pública

\section{REFERÊNCIAS BIBLIOGRÁFICAS}

ALMEIDA, C. M. (Coord.), 1989. Os Atalhos da Mudança na Saúde no Brasil: 9 Estudos de Caso. Rio de Janeiro: OPAS/OMS.

CAMPOS, F. E., 1988. Resolutividade: Uma Aproximação à Avaliação Qualitativa dos Serviços de Saúde. Tese de Doutorado, Rio de Janeiro: Escola Nacional de Saúde Pública, Fundação Oswaldo Cruz.

CARVALHO, F. M.; SILVANY-NETO, A. M.; PAIM, J. S.; MELO, A. M. C. \& AZARO, M. G. A., 1988. Morbidade referida e utilização de consulta médica cm cinco populações do Estado da Bahía. Ciência e Cultura, 40: 853-858.

FERNANDES SILVA, S.; SHIMITI, M.; GUIMARÃES, E. S. S. \& KNOTT, C., 1991. A proposta de SILOS $\mathrm{cm}$ uma cidade de porte médio. Saúde em Debate, 33: 96-100.

FORMIGLI, V.; COSTA, H.; MOLESINI, J. \& FERNANDES, R., 1992. Organização da Atenção à saúde em Distritos Sanitários na Bahia. Revista Baiana de Enfermagem, 5: 05-20.

FRENK, J. M., 1985. El concepto y la medición de accesibilidad. Salud Publica de Mexico, 27: 438-453.

HENDERSON, R. H. \& SUNDARESAN, T., 1982. Cluster sampling to assess immunization coverage: a review of experience with a simplified sampling method. Bulletin of the World Health Organization, 60: 253-260.

IBGE (Fundação Instituto Brasileiro de Geografía e Estatística), 1986. Pesquisa Nacional por Amostra Domiciliar. Rio de Janeiro: IBGE.

KADT, E. \& TASCA, R., 1993. Promovendo a Eqüidade. Um Novo Enfoque com Base no Setor da Saúde. São Paulo: Hucitec/Salvador: Cooperação Italiana em Saúde.

LEMESHOW, S. \& ROBINSON, D., 1985. Surveys to mensure programme coverage and impact: a review of the methodology used by the expanded programme on immunization. World Health Statistic Quarterly, 38: 65-75.

LEMME, A. C.; NORONHA, G.; RESENDE, J. B., 1991. A satisfação do usuário em Hospital Universitário. Revista de Saúde Pública, 25: 41-46.
LWANGA, S. K. \& LEMESHOW, S., 1991. Sample Size Determination in Health Studies. Geneva: WHO.

MENDES, E. V., 1991. O Consenso do Discurso e o Dissenso da Prática Social: Notas Sobre a Municipalização de Saúde no Brasil. São Paulo. (Mimeo.)

MINAYO, M. C. S., 1922. O Desafio do Conhecimento. Pesquisa Qualitativa em Saúde. São Paulo: Hucitec/ Rio de Janeiro: Abrasco.

MORAES, I. H. S. \& KALIL, M. E. X. (Coords.), 1993. Sistemas Locais de Saúde (SILOS): Bibliografia Comentada da Produção Brasileira. Rio de Janeiro: Escola Nacional de Saúde Pública (Pares)/Salvador: Cooperação Italiana.

MPAS (Ministério da Previdência e Assistência Social) \& CONASP (Conselho de Administração de Saúde Previdenciária), 1982. Parâmetros para Planejamento Assistencial a Serem Utilizados no Inamps, anexo à Portaría no. 3046 de 20 de julho de 1982. (Mimeo.)

MPAS (Ministério da Previdência e Assistência Social) \& INAMPS (Instituto Nacional de Assistência Médica da Previdência Social), 1987. Sistemas Unificados e Descentralizados de Saúde (SUDS) - Decreto no 94.657 de 20 de julho de 1987, exposiçã de motivos, 031, p. 3, 9.

NASCIMENTO, A., 1991. Municipalização: trinta anos de experiência e frustração. Radis Tema, 12: 03-06.

NEVES, G. H., 1986. Cambé e Baurú: Dois Casos de Municipalização. Rio de Janeiro: Instituto Brasileiro de Administração Municipal. (Série Experiências Inovadoras)

OMS (Organización Mundial de la Salud), 1981. Preparación de Indicadores para Vigilar los Progresos Realizados en el Logro de la Salud para Todos en el Año 2000. Genebra: OMS.

OPS (Organización Panamericana de la Salud) \& OMS (Organización Mundial de la Salud), 1990. Reunion Subregional sobre Evaluacion de los Sistemas Locales de Salud (SILOS) en los Países del cono Sur y Brasil. Salvador, 7 al 9 de noviembre, Relato Final. (Mimeo.)

PAIM, J. S., 1993. A reorganização das práticas em Distritos Sanitários. In: Distrito Sanitário. O Processo Social de Mudança das Práticas Sanitárias do Sistema Único de Saúde (E. V. Mendes, org.), pp. 187-220, São Paulo: Hucitec/Rio de Janeiro: Abrasco.

SES (Secretaria Estadual de Saúde, Distrito Sanitário de Pau da Lima), 1992. Avaliação - 1991. Salvador. (Mimeo.) 
SILVA, L. M. V., 1990. A Descentralização das Ações de Saúde no Município: O Caso de Camaçari. Tese de Doutorado, São Paulo: Departamento de Medicina Preventiva, Universidade de São Paulo.

SILVA, L. M. V. \& FORMIGLI, V. L., 1993. Avaliação e os Projetos de Reorganização das Práticas de Saúde: Conceitos e Abordagens. II Congresso Nacional da Rede IDA - Brasil. 09 a 12 de junho, São Paulo: Secretaria Nacional da Rede IDA - Brasil. (Mimeo.)

1994. Avaliação em saúde: limites e perpectivas. Cadernos de Saúde Pública, 10: 80-91.

SUS (Sistema Único de Saúde) \& DSPL (Distrito Sanitário de Pau da Lima, Bahia), 1991. Avaliação de Atividades. Salvador: DSPL. (Mimeo.)
1992. Avaliação de Atividades. Salvador:

DSPL. (Mimeo.)

1993. Avaliação de Atividades. Salvador: DSPL. (Mimeo.)

UNICEF (Fundo das Nações Unidas para a Infância); SOCEP; SESCE (Secretaria Estadual de Saúde do Ceará) \& HOPE II, 1990. Pesquisa de Saúde Materno Infantil no Ceará PESMIC-2. Comparação de Dois Estudos de Abrangência Estadual, 1987-90. Ceará. (Mimeo.) 